\title{
The Notch1/cyclooxygenase-2/Snail/E-cadherin pathway is associated with hypoxia-induced hepatocellular carcinoma cell invasion and migration
}

\author{
LING-XIANG YU $^{1 *}$, LIANG ZHOU ${ }^{2 *}$, MO LI $^{3 *}$, ZHI-WEI LI $^{1}$, DE-SHENG WANG ${ }^{2}$ and SHAO-GENG ZHANG ${ }^{1}$ \\ ${ }^{1}$ Department of Hepatobiliary Surgery, 302 Hospital of PLA, Beijing 100039; Departments of ${ }^{2}$ Hepatobiliary Surgery and \\ ${ }^{3}$ Orthopaedics, Xijing Hospital, The Fourth Military Medical University, Xi'an, Shaanxi 710032, P.R. China
}

Received July 23, 2012; Accepted September 3, 2012

DOI: $10.3892 /$ or.2012.2103

\begin{abstract}
Hepatocellular carcinoma (HCC) is one of the most common malignancies worldwide; however, the prognosis of HCC patients remains poor. This poor prognosis is mainly attributed to the high rate of intrahepatic and distant metastasis. HCC often occurs in a hypoxic environment and hypoxia can activate metastatic programs, ultimately leading to tumor recurrence or metastasis. Thus, the discovery and subsequent development of novel agents to block HCC invasion and migration are the primary objectives of hepatic cancer research. The Notch1 signaling pathway might be involved in hypoxiainduced carcinoma metastasis. However, the mechanisms by which Notch1 mediates cell metastasis, particularly in hepatocellular carcinoma, are not yet entirely clear. The results of the present study show that hypoxia increases the invasion and migration capacities of different HCC cells. Activation of the Notch1 signaling pathway contributes to hypoxia-induced invasion and migration in HCC cells. The activated Notch1 signaling pathway can regulate Snail/E-cadherin through cyclooxygenase-2 (COX-2) under hypoxic conditions. The above results suggest that the Notch1/COX-2/Snail/E-cadherin pathway is possibly associated with hypoxia-induced invasion and migration in HCC cells. Thus, targeting Notch1 may be useful for devising novel preventive and therapeutic strategies for HCC.
\end{abstract}

Correspondence to: Professor De-Sheng Wang, Department of Hepatobiliary Surgery, Xijing Hospital, The Fourth Military Medical University, Xi'an, Shaanxi 710032, P.R. China

E-mail: deshengwang_666@163.com

Professor Shao-Geng Zhang, Department of Hepatobiliary Surgery, 302 Hospital of PLA, Fengtai District West Fourth Ring Road No. 100, Beijing 100039, P.R. China

E-mail: zhangshaogeng302@yahoo.cn

*Contributed equally

Key words: Notch1, cyclooxygenase-2, Snail, E-cadherin, hypoxia, invasion, migration

\section{Introduction}

As a major type of primary liver cancer, HCC is one of the most common malignancies worldwide (1). Despite extensive research focusing on different therapies and on the molecular carcinogenesis of HCC, the prognosis of HCC patients remains poor. The majority of HCC patients eventually succumb to intrahepatic and distant metastases after resection or transplantation (2), therefore the discovery and subsequent development of novel agents to block HCC invasion and migration are the primary research objectives for HCC.

Solid tumors often occur in hypoxic environments due to the tumors' expansion and the inadequacy of their local vasculature. Robust tumor growth requires the presence of a local vascular network that supplies both oxygen and nutrients to tumor cells. However, highly proliferating tumor cells develop faster than the vasculature, and tumor cells can rapidly encounter an avascular environment deficient in oxygen (3). Upon histological examination, necrotic cells are often found in the central core of tumors, suggesting severe hypoxia and nutrient deprivation, resulting in cell death. Although the overall survival of most patients is prolonged after the obstruction of hepatic arterial blood flow, this success is transient without offering an enduring cure. It has been noted that hypoxia can kill most tumor cells, but it applies a strong selective pressure, resulting in the survival of the most aggressive and metastatic cells (4). Such cells can escape the noxious hypoxic microenvironment by activation of metastatic programs, ultimately leading to tumor recurrence or metastasis (3).

The disruption of normal cell-cell adhesion contributes to the metastasis of tumor cells $(5,6)$. This disruption can be achieved by decreasing cadherin or catenin family members or by activating some signaling pathways (5). Previous research has shown that reduction of E-cadherin is relevant to tumor invasion, metastasis, and unfavorable prognosis (7-9). Abnormalities in the expression of E-cadherin are frequently associated with invasiveness and lymph node or distant metastasis in a variety of human cancers, including HCC. Snail, a zinc finger transcription factor, has been described to contribute to repressing the transcription of the E-cadherin gene by binding to the E-boxes of the CDH1 promoter (10). Upregulation of Snail also correlates with metastasis and poor 
prognosis, whereas silencing of Snail is critical for reducing tumor growth and invasiveness $(11,12)$. In addition to breast, renal, pancreatic, and colon carcinoma, the changes of Snail/E-cadherin have been demonstrated in hypoxic hepatic tumor cells, which are correlated with increased migration and invasion capacities (13-15).

Studies have revealed that the Notch signaling pathway is not only involved in embryonic development and in cell fate determination, but that it also plays an important role in cancer $(16,17)$. Another study also showed that the Notch signaling pathway is an attractive mediator between hypoxia and tumor cells (18). The Notch signaling pathway includes Notch ligands, negative and positive modifiers, and Notch target transcription factors. Notch1, a Notch signaling pathway receptor, mRNA and protein expression are strong in $\mathrm{HCC}$, and significantly higher than adjacent non-tumor liver, and the expression of Notch1 is significantly different among differentiation grades (19). Notch1 has been shown to be upregulated in prostate cancer, small cell lung cancer, and pancreatic carcinoids. In contrast to its tumor-facilitative role, activation of the Notch1 signaling pathway was reported to inhibit cancer cell growth and induce apoptosis of B cells and other hematopoietic lineages in vitro $(20,21)$. A previous study showed Notch directly upregulated Snail expression by recruitment of the Notch intracellular domain to the Snail promoter and elevated the hypoxia-induced upregulation of lysyl oxidase (LOX), which stabilizes the Snail-1 protein (22). Another report demonstrated that N1ICD can oppose Snail-dependent HCC cell invasion by binding and inducing proteolytic degradation of Snail (23). However, Notch1 signaling plays a paradoxical role depending on the tissue type $(16,17)$. In this study, we investigated another potential mechanism by which the Notch1/ cyclooxygenase-2 (COX-2)/Snail/E-cadherin pathway is possibly involved in hypoxia-induced HCC cell invasion and migration.

\section{Materials and methods}

Cell culture and reagents. The human HCC cell lines HepG2, SMMC-7721 and MHCC97H were cultivated in RPMI-1640 medium supplemented with $10 \%$ fetal calf serum (Sigma Chemical Co., St. Louis, MO, USA). To attain normoxic conditions, the cultures were maintained at $37^{\circ} \mathrm{C}$ in a humidified incubator containing $20 \% \mathrm{O}_{2}, 5 \% \mathrm{CO}_{2}$, and $75 \% \mathrm{~N}_{2}$. For the hypoxic condition, cells were cultured and handled at $37^{\circ} \mathrm{C}$ in a Proox model 110 compact gas oxygen controller (Reming Bioinstruments, Redfield, NY, USA) flushed with a gas mixture of 3,2 or $1 \% \mathrm{O}_{2}, 5 \% \mathrm{CO}_{2}$ and 92,93 or $94 \%$ $\mathrm{N}_{2}$. The presence of $\mathrm{PO}_{2}$ in the culture medium was measured using a trace oxygen analyzer (Oxi 315i/set, WTW, Weilheim, Germany). Primary antibodies for Notch1, Hes1, E-cadherin, Snail, COX-2 and $\beta$-actin were purchased from Santa Cruz Biotechnology (Santa Cruz, CA, USA). Primary antibodies for the Notch1 receptor intracellular domain (N1ICD) were purchased from Abcam (Cambridge, UK). All secondary antibodies were obtained from Pierce (Rockford, IL, USA). Notch1 small interfering RNA (siRNA), Snail siRNA, and siRNA control were obtained from Santa Cruz Biotechnology. Lipofectamine $^{\mathrm{TM}} 2000$ was purchased from Invitrogen (Carlsbad, CA, USA). To suppress endogenous COX-2 activity, $50 \mu \mathrm{mol} / 1 \mathrm{NS}-398$ (Sigma-Aldrich) in DMSO were added.
Prostaglandin $\mathrm{E}_{2}\left(\mathrm{PGE}_{2}\right.$; Sigma-Aldrich) in ethanol was used at $10 \mu \mathrm{g} / \mathrm{ml}$. All other chemicals and solutions were purchased from Sigma-Aldrich, unless otherwise indicated.

Growth curves and measuring cell growth. The HepG2 and MHCC97H cells were seeded into 6-well cell culture plates at a density of $5 \times 10^{3}$ cells/well and were grown for $\leq 6$ days in normoxic and hypoxic conditions. A hemocytometer was used every day to determine the number of cells. We used the relative density of the cells (versus the density of the primary cells at $100 \%$ ) to establish the growth curve. Each experiment included six replications and was repeated three times. The data are summarized as the means \pm SD.

RNA isolation and reverse transcription polymerase chain reaction ( $R T-P C R)$. Total-RNA was extracted from the cells using the TRIzol reagent, according to the manufacturer's instructions (Invitrogen). Reverse transcription of total cellular RNA was performed using the one-step RT-PCR kit (MBI Fermentas, Vilnius, Lithuania) according to the manufacturer's instructions. The polymerase chain reaction (PCR) primers used were: 5'-CCGTCATCTCCGACTTCATCT-3' (forward) and 5'-GTGTCTCCTCCCTGTTGTTCTG-3' (reverse) for Notch1; 5'-TCAACACGACACCGGATAAA-3' (forward) and 5'-CCGCGAGCTATCTTTCTTCA-3' (reverse) for Hes1; 5'-TC CCATCAGCTGCCCAGAAA-3' (forward) and 5'-ATTGTCC TTGTGTCCTCAGT-3' (reverse) for E-cadherin; 5'-TTCTTCT GCGCTACTGCTGCG-3' (forward) and 5'-AGAAGGAGA GGTATGGACGGG-3' for Snail; and 5'-AGCGGGAAATCGT GCGTG-3' (forward) and 5'-CAGGGTACATGGTGGTGCC-3' (reverse) for $\beta$-actin. The PCR conditions were: after initial denaturation at $94^{\circ} \mathrm{C}$ for $4 \mathrm{~min}, 30$ cycles of denaturation at $94^{\circ} \mathrm{C}$ for $45 \mathrm{sec}$, annealing at each appropriate temperature as described for $30 \mathrm{sec}$ and extension at $72^{\circ} \mathrm{C}$ for $45 \mathrm{sec}$. PCR products were separated by electrophoresis on $1 \%$ agarose gel and were visualized with ethidium bromide staining. Gene expression was presented as the relative yield of the PCR product from target sequences compared to that of the $\beta$-actin gene. The mean values from three independent experiments were taken as the results.

Small interfering RNA transfection. According to the protocol of Lipofectamine 2000, HepG2 and MHCC97H cells were transfected with Notch1 siRNA, Snail siRNA, and siRNA control. The cells were allowed to grow for $48 \mathrm{~h}$ and were then harvested for further analysis.

Protein extraction and western blotting. The cells were lysed in lysis buffer (50 mmol/l Tris (pH 7.5), $100 \mathrm{mmol} / \mathrm{l} \mathrm{NaCl}$, $1 \mathrm{mmol} / \mathrm{l}$ EDTA, $0.5 \% \mathrm{NP} 40,0.5 \%$ Triton X-100, $2.5 \mathrm{mmol} / \mathrm{l}$ sodium orthovanadate, $10 \mu \mathrm{l} / \mathrm{ml}$ protease inhibitor cocktail, and $1 \mathrm{mmol} / \mathrm{l} \mathrm{PMSF}$ ) by incubating for $20 \mathrm{~min}$ at $4^{\circ} \mathrm{C}$. The protein concentration was determined using the Bio-Rad assay system (Bio-Rad, Hercules, CA, USA). Total proteins were fractionated using SDS-PAGE and were transferred onto nitrocellulose membranes. The membranes were blocked with $5 \%$ non-fat dried milk or bovine serum albumin in $1 \mathrm{X}$ TBS buffer containing $0.1 \%$ Tween-20 and were then incubated with the appropriate primary antibodies. Horseradish peroxidase-conjugated anti-rabbit or anti-mouse IgG was used as 
the secondary antibody, and the protein bands were detected using the enhanced chemiluminescence detection system (Amersham Pharmacia Biotech). Quantification of western blot analyses was performed using laser densitometry, and the results are presented as the means of three independent experiments with error bars representing SD.

MTT assay. The differently treated cells were seeded into 96-well cell culture plates at a density of $1 \times 10^{4}$ cells/well and were grown for $\leq 48 \mathrm{~h}$ in hypoxic conditions $\left(3 \% \mathrm{O}_{2}\right)$. Cell viability was assessed using the 3-(4,5-dimethyl-2-thiazolyl)2,5-diphenyl-2H-tetrazolium bromide (MTT) assay (Sigma Chemical Co.) according to the manufacturer's protocol. Each experiment included six replications and was repeated three times. The data are summarized as the means \pm SD.

Flow cytometry for the analysis of cell apoptosis. To determine the number of apoptotic cells, Annexin V assays were performed using an apoptosis detection kit (Annexin V-FITC/ propidium iodide (PI) Staining kit; Immunotech Co., Marseille, France). Briefly, 1.5x10 ${ }^{5}$ HepG2 and MHCC97H cells were transfected with Notch1 siRNA, Snail siRNA, and siRNA control and were plated in 24-well plates under hypoxic conditions. After $48 \mathrm{~h}$, the cells were harvested, washed in cold PBS, incubated for $15 \mathrm{~min}$ with fluorescein-conjugated Annexin V and PI, and analyzed using flow cytometry. PI-negative and Annexin V-positive cells were considered to be early apoptotic, while cells that were both PI- and Annexin V-negative were considered non-apoptotic.

Migration and invasion assays. Cell migration was analyzed using non-matrigel-coated transwell cell culture chambers (8- $\mu \mathrm{m}$ pore size) (Millipore, Billerica, MA, USA). Cell invasion was analyzed using matrigel-coated transwell cell culture chambers ( $8-\mu \mathrm{m}$ pore size) (Millipore). Briefly, differently treated cells were serum-starved for $24 \mathrm{~h}$ and were plated in the upper insert at $5 \times 10^{4}$ cells per ml for a 24 -well chamber in serum-free medium. Medium containing $10 \%$ serum as a chemoattractant was added to the well. The cells were incubated in normoxic or hypoxic conditions for $24 \mathrm{~h}$. Non-invading cells were removed from the upper surface by scrubbing with a cotton swab, after which the membrane was fixed with $4 \%$ formaldehyde for $10 \mathrm{~min}$ at room temperature and was stained with $0.5 \%$ crystal violet for $10 \mathrm{~min}$. Finally, invasive cells were counted at magnification $\mathrm{x} 200$ from 10 different fields of each filter. For treatment with NS-398 or $\mathrm{PGE}_{2}$, the cells were pretreated for $2-4 \mathrm{~h}$, and the treatment continued during the invasion experiment.

Statistical analysis. Each experiment was repeated at least three times. All data were summarized and are presented as the means \pm SD. The differences among means were statistically analyzed using a t-test. All statistical analyses were performed using SPSS 13.0 software (Chicago, IL, USA). $\mathrm{P}<0.05$ was considered to indicate statistically significant differences.

\section{Results}

Snail/E-cadherin is involved in hypoxia-induced invasion and migration of HCC cells. Tumor hypoxia is associated with enhanced invasion and migration. To investigate this process in hepatocellular carcinoma, we analyzed the invasion and migration capacities in HepG2, SMMC-7721 and MHCC97H cells cultivated in hypoxic conditions. As shown in Fig. 2A-C, we found that under hypoxic conditions dead cells would increase significantly in, at most, 4 days and that increasingly more cells would die with the amount of $\mathrm{PO}_{2}$ decreasing. Therefore, in this experiment, we only cultured the cells for $48 \mathrm{~h}$ in hypoxic conditions, with $3 \% \mathrm{O}_{2}$ to mimic hypoxic conditions. In normoxic conditions, the three cell lines showed different invasion and migration capacities. Using transwell cell culture chambers, we found that, in the three cell lines, the level of penetration was lowest in the HepG2 cells and highest in the MHCC97H cells (Fig. 1A and B). As illustrated in Fig. 1A and B, the three cell lines showed higher levels of penetration through transwell cell culture chambers, with or without matrigel coating in hypoxic conditions versus normoxic conditions. However, comparing the degrees of increased penetration, HepG2 was the highest, and $\mathrm{MHCC} 97 \mathrm{H}$ was the lowest of the threes cell lines. These results demonstrate that hypoxia can increase the invasion and migration capacity in HCC cells.

There was an inverse correlation between the expressions of Snail and E-cadherin in the three cell lines. With RT-PCR and western blotting, E-cadherin was downregulated, and Snail was upregulated in mRNA and protein levels in the three cell lines cultured in hypoxic conditions (Fig. 1C and D). However, the variation in amplitude of E-cadherin or Snail exhibited significant differences in the three HCC cell lines. With the invasion and migration capacities of HCC cells increasing, the variation in amplitude of E-cadherin or Snail decreased. Particularly in MHCC97H cells, the changes in E-cadherin and Snail were slight. However, these results indicated that hypoxia was able to downregulate expression of E-cadherin and upregulate expression of Snail. As shown in Fig. 1E and F, Snail siRNA-transfected cells showed a low level of penetration through the membrane, compared with control siRNA-transfected cells in hypoxic conditions. These results indicated that Snail/E-cadherin was involved in hypoxia-induced invasion and migration of HCC cells.

Suppression of Notchl can reduce hypoxia-induced invasion and migration of HCC cells by regulating Snail/E-cadherin. To address whether hypoxia potentiated the Notch1 signaling pathway, we examined the effect of hypoxia on the Notch1 signaling pathway in HepG2, SMMC-7721 and MHCC97H cells. RT-PCR analysis showed that the mRNA expressions of Notch1 receptor and the downstream target gene Hes1 in the three HCC cell lines increased under hypoxic conditions, compared with normoxic conditions (Fig. 3A). Notch1 receptor, N1ICD and Hes1 also exhibited similar increased tendencies (Fig. 3B) in protein levels. The variation in amplitude of the Notch1 signaling pathway also exhibited significant differences. In MHCC97H cells, the change in Notch1 was slight. However, in the three cell lines, N1ICD and Hes1 were upregulated in hypoxic conditions. Therefore, these results illustrate that the Notch1 signaling pathway is activated in hypoxic conditions. The invasion and migration capacities were lowest in HepG2 cells and were highest in MHCC97H cells. Therefore, in the following experiment, we only used 
A
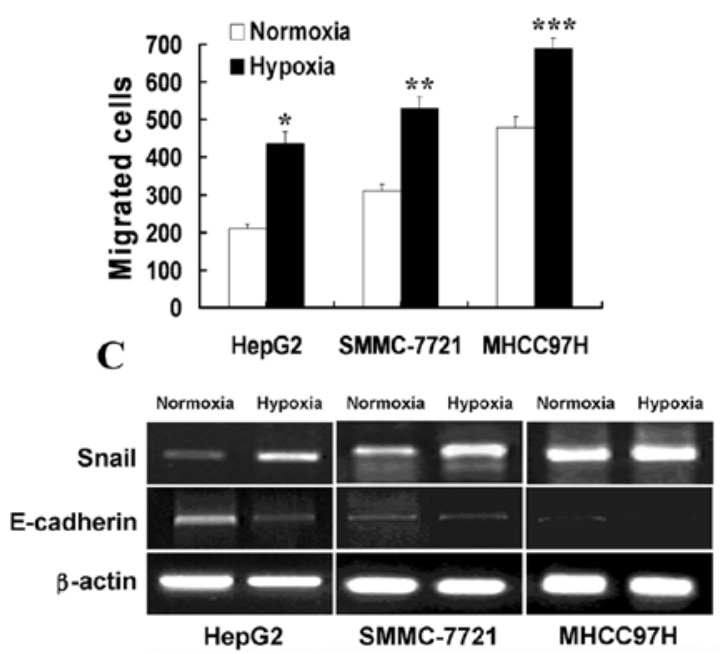

$\mathrm{E}$

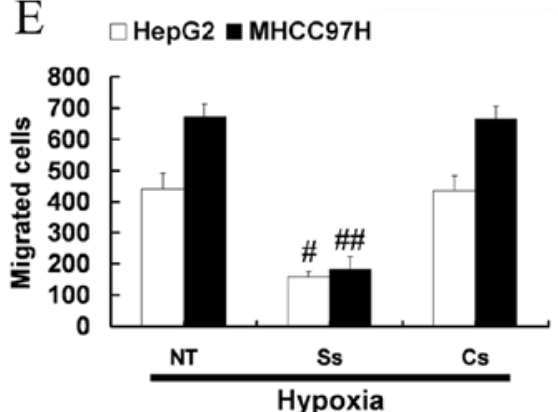

B
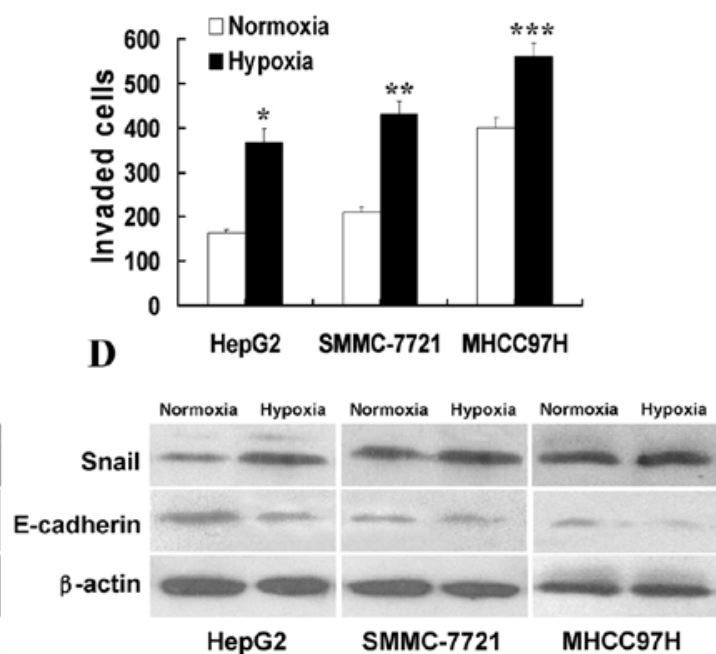

$\mathrm{F}$

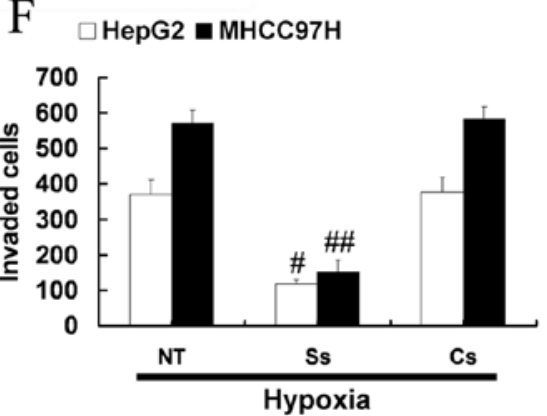

Figure 1. Snail/E-cadherin participated in hypoxia-induced invasion and migration of HCC cells. (A and B) Using transwell cell culture chambers, the invasion and metastasis capacities of HCC cells were detected in normoxic $\left(20 \% \mathrm{O}_{2}\right)$ and hypoxic conditions $\left(3 \% \mathrm{O}_{2}\right)$ after $48 \mathrm{~h}$. (C and D) In normoxic and hypoxic conditions, the mRNA and protein expressions of Snail and E-cadherin were detected in HCC cells. (E and F) Using transwell cell culture chambers, the invasion and migration capacities of different treated HepG2 and MHCC97H cells were detected. Non-transfected and control siRNA-transfected cells were used as control. NT, non-transfection; Ss, Snail siRNA-transfection; Cs, control siRNA-transfection. Data represent the means \pm SD; ${ }^{\text {P }<0.05 ~ c o m p a r e d ~ w i t h ~ H e p G 2 ~}$ cells cultivated in normoxic conditions; ${ }^{* *} \mathrm{P}<0.05$ compared with $\mathrm{SMMC}-7721$ cells cultivated in normoxic conditions; ${ }^{* * *} \mathrm{P}<0.05$ compared with $\mathrm{MHCC} 97 \mathrm{H}$ cells cultivated in normoxic conditions; ${ }^{\#} \mathrm{P}<0.05$ compared with $\mathrm{Cs}$ in HepG2 cells; ${ }^{\# \#} \mathrm{P}<0.05$ compared with Cs in MHCC97H cells.
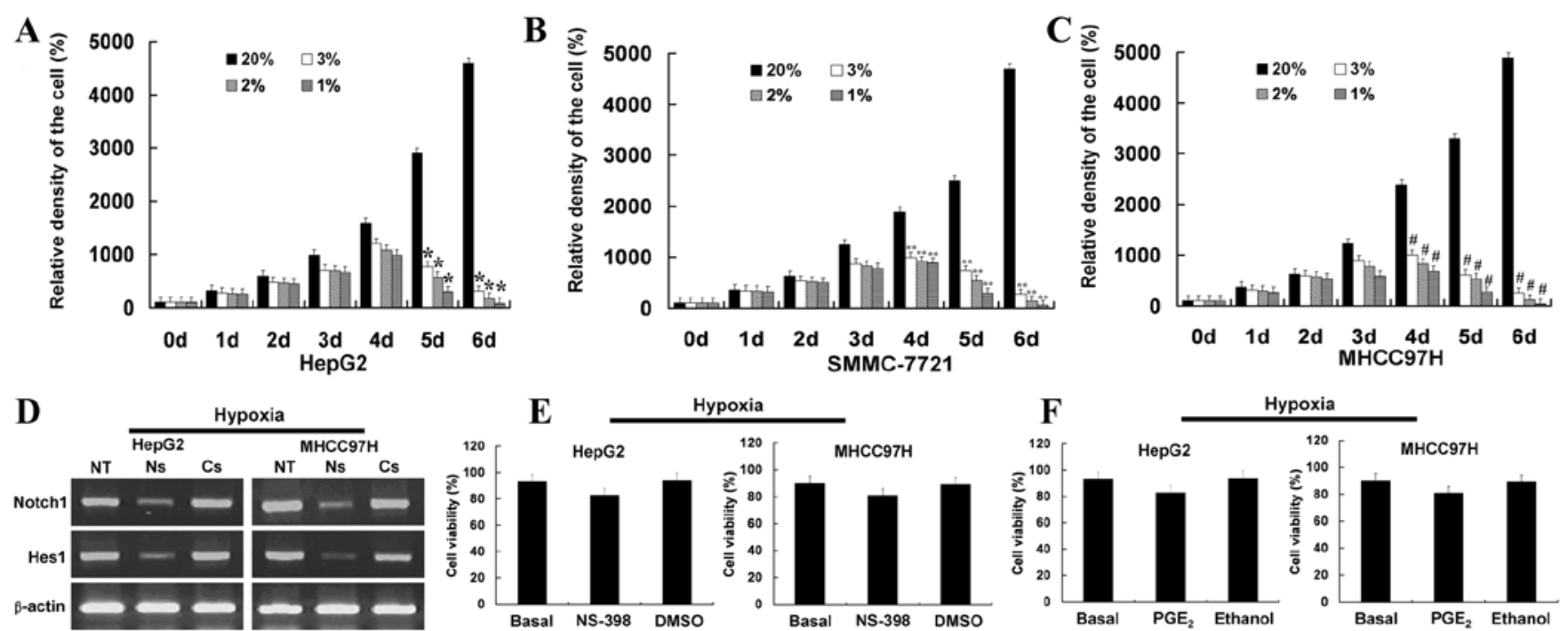

Figure 2. (A-C) HCC cells cannot survive in hypoxic conditions for a long time. Three $\mathrm{HCC}$ cell lines were cultivated with various concentrations of $\mathrm{O}_{2}$ for 6 days Cell density was measured every day using a hemocytometer. Initial cell density was used as control. Data represent the means $\pm \mathrm{SD}$; ${ }^{*} \mathrm{P}<0.05$ compared with the HepG2 cells cultivated in normoxic conditions $\left(20 \% \mathrm{O}_{2}\right) ;{ }^{* *} \mathrm{P}<0.05$ compared with the SMMC-7721 cells cultivated in normoxic conditions; ${ }^{*} \mathrm{P}<0.05$ compared with the MHCC97H cells cultivated in normoxic conditions. (D) In different treated HepG2 and MHCC97H cells cultivated in hypoxic conditions, RT-PCR measured the mRNA expressions of Notch1 and Hes1. NT, non-transfection; Ss, Snail siRNA-transfection; Cs, control siRNA-transfection. (E and F) Neither 50 $\mu$ mol/1 NS-398 nor $10 \mu \mathrm{g} / \mathrm{ml} \mathrm{PGE}_{2}$ affected cell viability. HepG2 and MHCC97H cells treated with $50 \mu$ mol/1 NS-398 or $10 \mu \mathrm{g} / \mathrm{ml}_{\mathrm{PGE}}$ were cultivated in hypoxic conditions after $48 \mathrm{~h}$. Cell viabilities were gauged by MTT assay. Basal cells (non-treated cells) and cells treated with DMSO or ethanol were used as control. 

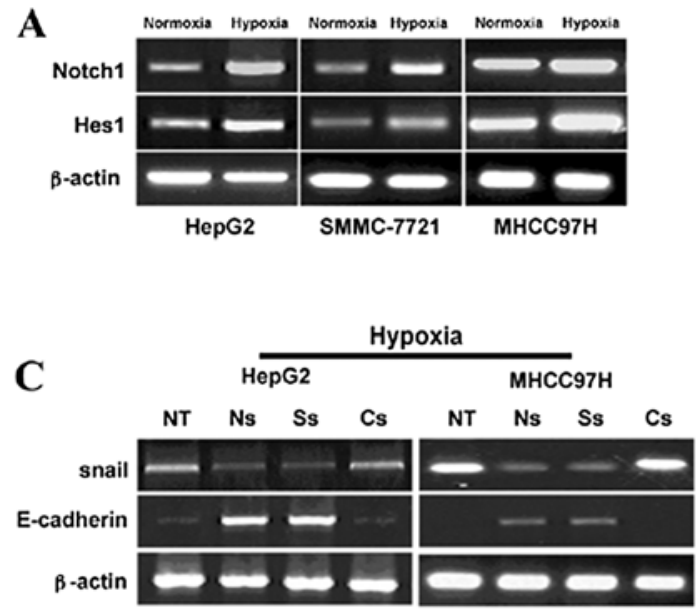
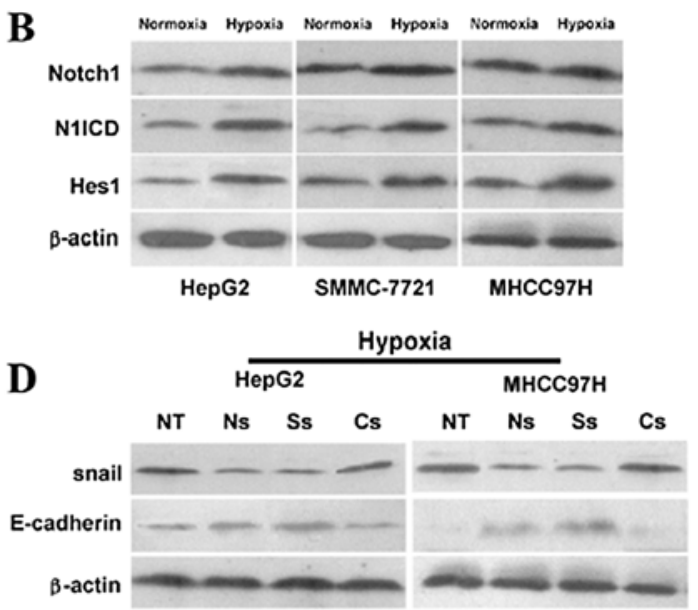

$\mathbf{E}$

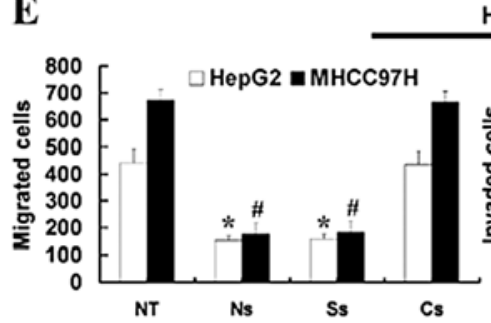

Hypoxia

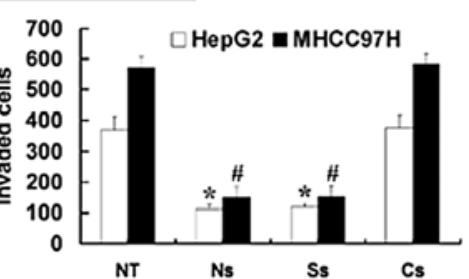

$\mathbf{F}$

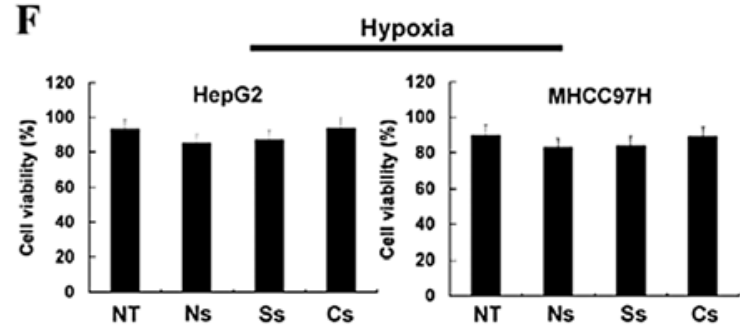

G

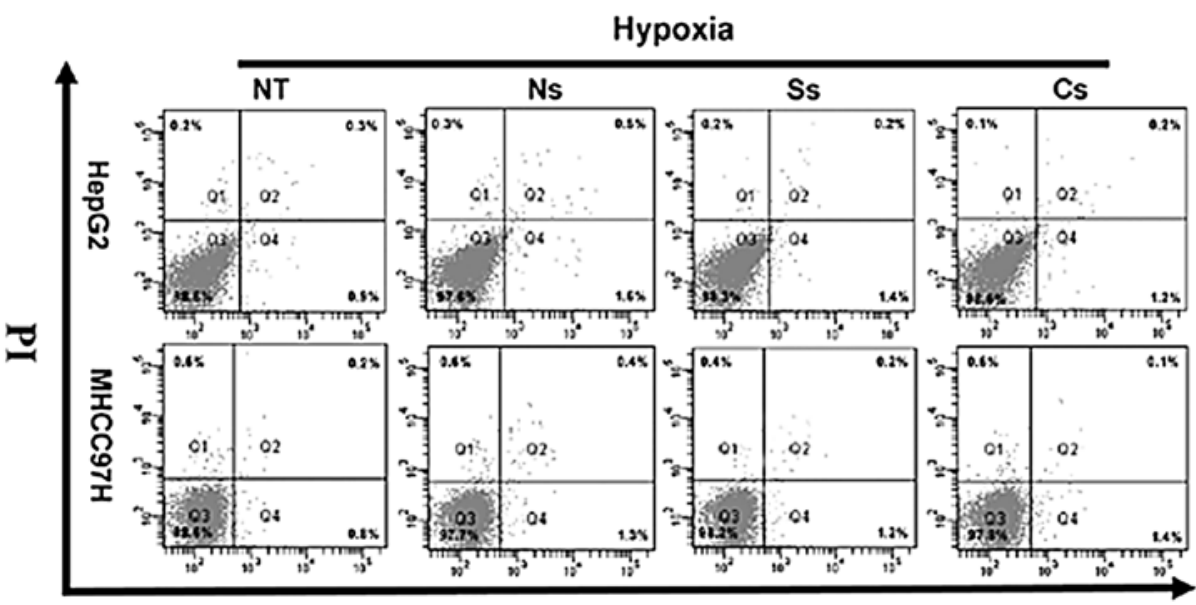

Annexin.V

Figure 3. Suppression of Notch1 can reduce hypoxia-induced invasion and migration of HCC cells through regulating Snail/E-cadherin. (A and B) In normoxic and hypoxic conditions, the mRNA expressions of Notch1 and Hes1 were measured by RT-PCR; the protein expressions of Notch1, N1ICD and Hes1 were measured by western blotting. (C and D) Using RT-PCR and western blotting, the mRNA and protein expressions of Snail and E-cadherin were measured in different treated HepG2 and MHCC97H cells cultivated in hypoxic conditions. (E) Using transwell cell culture chambers, the invasion and metastasis capacities were measured in different treated HepG2 and MHCC97H cells cultivated in hypoxic conditions. (F and G) Different treated HepG2 and MHCC97H cells were cultivated in hypoxic conditions after $48 \mathrm{~h}$. Cell viabilities were gauged by MTT assay and flow cytometry. Non-transfected and control siRNAtransfected cells were used as control. Data represent the means \pm SD, NT, non-transfection; Ns, Notch-1 siRNA-transfection; Ss, Snail siRNA-transfection; Cs, control siRNA-transfection. ${ }^{*} \mathrm{P}<0.05$ compared with Cs in HepG2 cells; ${ }^{\#} \mathrm{P}<0.05$ compared with Cs in MHCC $97 \mathrm{H}$ cells.

HepG2 and MHCC97H cells. To further examine whether the Notch1 signaling pathway was involved in regulating invasion and migration, HepG2 and MHCC97H cells were transfected with human Notch1 siRNA, Snail siRNA or control siRNA and were cultivated in hypoxic conditions. The cells transfected with Notch1 siRNA showed low expressions of Notch1 and Hes1 mRNA, as confirmed by RT-PCR (Fig. 2D). We also detected the expressions of Snail and E-cadherin in mRNA and the protein levels in these siRNA-transfected cells cultured in hypoxic conditions. As shown in Fig. 3C and D, Notch1 siRNA and Snail siRNA were able to downregulate expression of Snail or upregulate expression of E-cadherin in mRNA and protein levels. Between both siRNA-transfected cells, mRNA and protein expressions of Snail or E-cadherin had no difference in changes. As illustrated in Fig. 3E, Notch1 siRNA-transfected cells showed low levels of penetration through the membrane, 

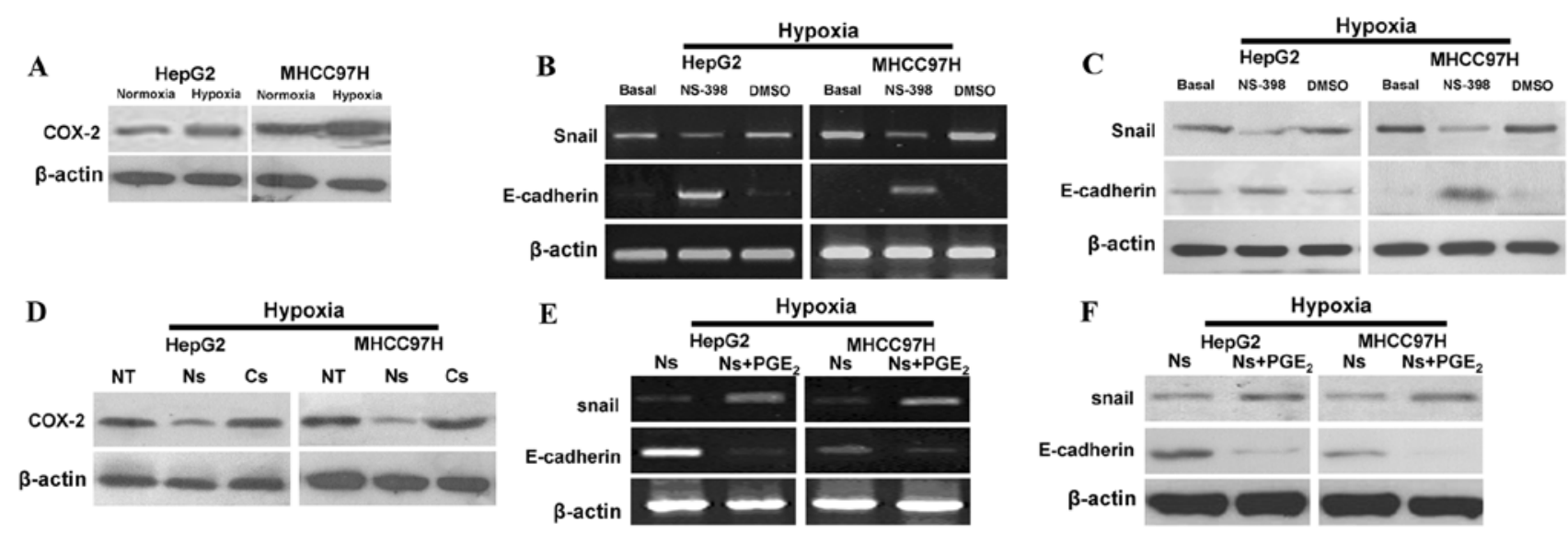

G

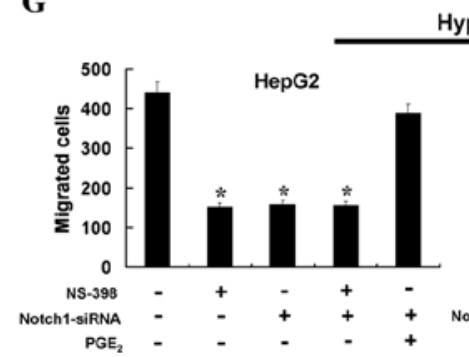

Hypoxia

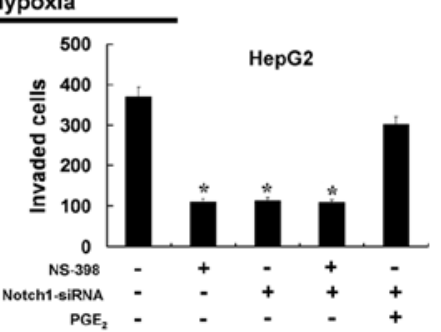

H

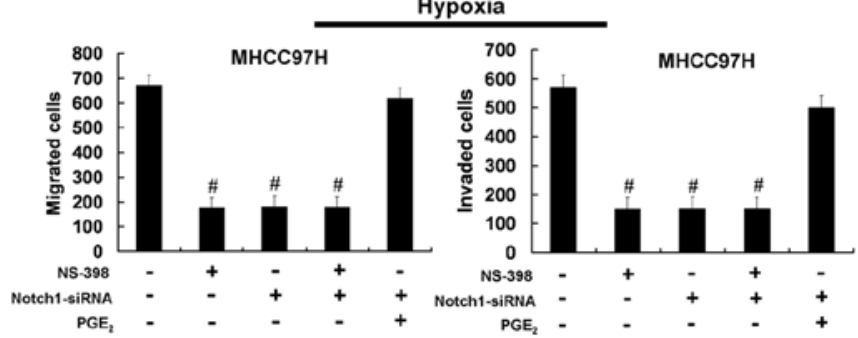

Figure 4. Suppression of Notch1 could inactivate Snail by regulating COX-2 resulting in the inhibition of invasion and migration in hypoxic conditions. (A) Using western blotting, the protein expressions of COX-2 were measured in HepG2 and MHCC97H cells cultivated in normoxic and hypoxic conditions. (B and C) Using RT-PCR and western blotting, the mRNA and protein expressions of Snail and E-cadherin were measured in different treated HepG2 and MHCC97H cells. Basal cells (non-treated cells) and cells treated with DMSO were used as control. (D) Using western blotting, the protein expressions of COX-2 were measured in different treated HepG2 and MHCC97H cells cultivated in hypoxic conditions. Non-transfected and control siRNA-transfected cells were used as control. (E and F) Using RT-PCR and western blotting, the mRNA and protein expressions of Snail and E-cadherin were measured in different treated HepG2 and MHCC97H cells cultivated in hypoxic conditions. ( $\mathrm{G}$ and $\mathrm{H}$ ) Using transwell cell culture chambers, invasion and metastasis capacities were measured in HepG2 and MHCC97H cells treated with Notch1-siRNA and/or $50 \mu$ mol/1 NS-398 and/or $2 \mu \mathrm{g} / \mathrm{ml} \mathrm{PGE}_{2}$ in hypoxic conditions. Data represent the means $\pm \mathrm{SD} ;{ }^{*} \mathrm{P}<0.05$ compared with non-treated HepG2 cells; ${ }^{*} \mathrm{P}<0.05$ compared with non-treated MHCC97H cells. NT, non-transfection; Ns, Notch-1 siRNA-transfection; Cs, control siRNA-transfection.

compared to control siRNA-transfected cells. Between Notch1 siRNA-transfected and Snail siRNA-transfected cells, the decreased levels of invasion and migration capacities showed no differences. In normoxic conditions, Notch1 siRNA-transfected cells also showed low levels of penetration through the membrane, compared to control siRNA-transfected cells (data not shown). To confirm that the decreased invasion and migration capacity was not due to apoptosis, we used an MTT assay and flow cytometry to detect different treated HCC cells. As the results show, Notch1 siRNA or Snail siRNA did not affect cell viability at $48 \mathrm{~h}$ in hypoxic conditions (Fig. $3 \mathrm{~F}$ and $\mathrm{G}$ ). The above-mentioned results indicate that suppression of Notch1 can reduce hypoxia-induced invasion and migration by regulating Snail/E-cadherin. In hypoxic conditions, suppression of Notch1 could inactivate Snail/E-cadherin by regulating COX-2, resulting in the inhibition of $\mathrm{HCC}$ cell invasion and migration.

To explore the potential mechanisms by which the Notch1 signaling pathway regulates Snail/E-cadherin, we focused on COX-2. Western blot analysis showed that hypoxia increased the protein expressions of COX-2 in HepG2 and $\mathrm{MHCC} 97 \mathrm{H}$ cells (Fig. 4A). To address whether hypoxia-induced changes of Snail/E-cadherin occurs through COX-2, HepG2 and MHCC97H cells were treated with COX-2 inhibitor NS-398 to block COX-2 activity. As shown in Fig. 4B and C, NS-398 can upregulate expression of E-cadherin and downregulate expression of Snail in mRNA and protein levels under hypoxic conditions. In the following experiment, we detected the protein expression of COX-2 in Notch1 siRNA-transfected cells cultured in hypoxic conditions. As shown in Fig. 4D, inhibition of Notch1 abrogated hypoxia-induced COX-2 expression. In normoxic conditions, COX-2 also showed a lower expression in Notch1 siRNA-transfected cells than in control siRNA-transfected cells (data not shown). In hypoxic conditions, treating Notch1 siRNA-transfected cells with exogenous $\mathrm{PGE}_{2}$, we found $\mathrm{PGE}_{2}$ can upregulate expression of Snail and downregulate expression of E-cadherin in mRNA and protein levels (Fig. 4E and F). To further study the relationship between COX-2 activity and Notch1 in the control of HCC invasion and migration in hypoxic conditions, we treated cells with NS-398 and Notch1 siRNA to block COX-2 activity and the Notch1 signal pathway, respectively. Treatment with NS-398 or Notch1 siRNA alone reduced the invasion and migration capacities of HepG2 and MHCC 97H cells. However, treatment with NS-398 in combination with Notch1 siRNA did not block these biological functions of HCC cells more than treatment with NS-398 or Notch1 siRNA alone. The suppressed capacity of invasion and migration by Notch1 knockdown was reversed after treatment with PGE2 in HCC cells (Fig. 4G and H). Using MTT assay, $50 \mu \mathrm{mol} / 1 \mathrm{NS}-398$ or $2 \mu \mathrm{g} / \mathrm{ml}$ PGE2 did not affect cell viability (Fig. 2E and F). 


\section{Discussion}

The results of the present study show that the activation of the Notch1 signaling pathway contributes to hypoxia-induced invasion and migration in HCC. The induction of Snail/Ecadherin is one of the invasive mechanisms during hypoxia. The activated Notch1 signaling pathway can regulate the Snail/E-cadherin through COX-2. These results supplement the invasion and migration mechanisms in $\mathrm{HCC}$ and further confirm the importance of the Notch1 signaling pathway in $\mathrm{HCC}$ under hypoxic conditions.

In the present study, we showed that, in hypoxic conditions, the capacities for invasion and migration in different HCC cell lines was enhanced. These results are also in accordance with previous studies $(14,15)$. As our results show, HCC cells cannot survive in hypoxic conditions for a long time, and they may explain why multiple strategies for the obstruction of hepatic arterial blood flow have achieved pronounced therapeutic effects for HCC, particularly unresectable HCC $(24,25)$. However, tumor hypoxia is associated with enhanced invasion, angiogenesis and distant metastasis $(26,27)$. Hypoxia can also activate some metastatic programs and allow tumor cells to escape from noxious hypoxic microenvironments, resulting in distant metastasis.

Changes of Snail/E-cadherin are most important in the hypoxia-induced metastasis of cancer (28). The loss of E-cadherin expression and the disassembly of E-cadherin adhesion plaques on the cell surface enable tumor cells to disengage from the primary mass and to move to conduits of dissemination (29). E-cadherin is also a critical factor in the process of the intrahepatic metastasis of HCC (30). Snail, a zinc finger transcription factor, has been described to contribute to repressing the transcription of the E-cadherin gene (10). Extensive studies have shown Snail is involved in cancer development and metastasis $(31,32)$. There have been strong inverse correlations between Snail and E-cadherin expression in panels of epithelial and dedifferentiated cells derived from carcinomas of various etiologies, including oral squamous carcinoma, breast, pancreas, colon, and bladder cancer, melanoma, fibroblasts and HCC (33-36).

Our data demonstrated similar conclusion to the above. In MHCC 97H cells cultured under hypoxic conditions, the changes in E-cadherin and Snail were slight. The reason for this finding may be that MHCC $97 \mathrm{H}$ is a type of high-invasion and high-migration HCC cell. To adapt its high-invasion and high-migration capacity, to normoxic conditions, Snail exhibited a high level, resulting in downregulated E-cadherin. The cells were in a balanced condition, and stimulation can affect signaling pathways or factors only to a limited extent. If the changes were beyond this range, they affected cellular function, and even caused cell death. Hypoxia can affect the expression of E-cadherin or Snail in HCC cells. However, MHCC97H cells exhibited a high expression of Snail in normoxic conditions, and hypoxia can induce changes of Snail that are also slight. This result may be caused by the cells' selfregulation mechanisms, which can protect cells and keep them from being damaged. However, by downregulating expression of Snail, the capacity of invasion and migration significantly decreased in HCC cells cultured in hypoxic condition. Thus, these data indicate that Snail/E-cadherin is involved in hypoxia induced invasion and migration. However, the mechanism of hypoxia-induced changes of Snail/E-cadherin in HCC is not clear.

As has been well established, the Notch signaling pathway is involved in the carcinogenesis, progress, invasion and neurovascular formation of numerous malignant tumors (37-39). However, knowledge of the role of Notch1 in hypoxia-induced invasion and migration of HCC is limited. The present study showed that hypoxia can activate the Notch1 signaling pathway. Expression of the Notch1 signaling pathway in MHCC97H cells increased substantially less than that in other cell lines, perhaps due to $\mathrm{MHCC} 97 \mathrm{H}$ cells having the greatest metastatic potential of the three cell lines. Due to the high invasiveness of MHCC 97H cells, under normoxic conditions, the Notch1 signaling pathway was highly activated. Overexpression of Notch1 was able to inhibit the growth of HCC cells (40). Thus, to avoid hypoxia-induced cell injury, Notch1 will not considerably change in MHCC97H cells. Increases of N1ICD and HES1 may have been caused by other reasons, such as increased ligands or some regulated factors of Notch1. The data also indicated that the signaling pathway has great multiplicity and that research into a single signaling pathway has been mistaken. However, due the current technical limitations of the methodology, multi-channel complex network research has been very difficult. This deficit was also a limitation of our research.

The notch signaling pathway is required to convert hypoxic stimuli into Snail/E-cadherin, increased motility, and invasiveness in different tumor cells (22). It is not clear whether Notch1 mediates hypoxia-induced changes of Snail/ E-cadherin in HCC cells. In our study, downregulated Notch1 downregulated expression of Snail and upregulated expression of E-cadherin in hypoxic condition. Downregulated Notch1 could also decrease the invasion and migration of HCC cells cultured in hypoxic conditions. The inhibitive degree of downregulated Notch1 was similar to downregulated Snail. However, the above results confirmed that Notch1 was involved in hypoxia-induced invasion and migration through regulating Snail/E-cadherin in HCC cells.

Sahlgren et al (22) demonstrated some molecular mechanisms coupling the Notch signaling pathway to Snail/E-cadherin and metastasis in hypoxic conditions. However, the physiological functions of cells are complex. The question remained whether another mechanism was involved in the abovementioned process. We focused on COX-2. Tumor COX-2 and its metabolite, prostaglandin $\mathrm{E}_{2}\left(\mathrm{PGE}_{2}\right)$, play important roles in regulating diverse cellular functions under physiological and pathological conditions (41-43). COX-2 is also overexpressed in a variety of malignancies, including colon, gastric, esophageal, prostate, pancreatic, breast, and lung carcinomas $(41,42,44,45)$. Elevated COX-2 expression is often associated with invasion and metastasis in lung and breast cancer $(46,47)$. COX-2/PGE ${ }_{2}^{-}$ dependent pathways contribute to the modulation of E-cadherin expression through $\mathrm{PGE}_{2}$ exposure, leading to enhanced Snail binding at the chromatin level (48). This relationship may be the mechanism by which COX-2 is involved in the invasion and metastasis of tumors. One study showed that Notch1 can regulate COX-2 expression in cancer through N1ICD bound to a COX-2 promoter (49). In the present study, we showed that Notch1 can also regulate Snail/E-cadherin in HCC cells in 
hypoxic conditions. Therefore, we hypothesized that in HCC, Notch1 might be involved in hypoxia-induced metastasis by regulating COX-2. We discovered that elevated COX-2 expression was found in $\mathrm{HCC}$ cells cultured in hypoxic conditions. Inhibition of COX-2 can invert hypoxia-induced upregulation of Snail and downregulation of E-cadherin. These results were similar to previous studies $(48,50)$ and also indicated that COX-2 can regulate hypoxia-induced change of Snail/E-cadherin in HCC. Markedly, downregulation of Notch1 can dramatically decrease the expression of COX-2 and downregulation of Notch1 or COX-2 had the same role in suppressing hypoxiainduced invasion and migration in HCC cells. However, exogenous $\mathrm{PGE}_{2}$ can inverse the role of downregulated Notch1 in regulating the expression of Snail/E-cadherin and invasion or migration of HCC cells in hypoxic conditions. These results can be explained by inhibited Notch1 causing COX-2/PGE to decrease and by the further decreased expression of Snail, resulting in E-cadherin upregulation. Exogenous $\mathrm{PGE}_{2}$ can also increase expression of Snail (48), so the role of downregulated Notch1 was neutralized. These results further indicate that Notch1 might be involved in hypoxia-induced invasion and migration by regulating $\mathrm{COX}-2$.

The above results suggest that the Notch1/COX-2/Snail/Ecadherin pathway possibly contributes to hypoxia-induced tumor invasion and migration. These findings also supplement the mechanisms of hypoxia-induced invasion and migration in HCC. According to the data, we presented experimental evidence that supports the anti-invasion and anti-migration effects of inhibited Notch1 in HCC. COX-2 inhibitors also have a promising role in the prevention and treatment of cancer despite their substantial gastrointestinal toxicity and increased cardiovascular risk $(51,52)$. Therefore, targeting Notch1 in combination with COX-2 inhibitors in lower doses may be useful for devising novel preventive and therapeutic strategies for HCC.

\section{References}

1. Yu MC, Yuan JM, Govindarajan S and Ross RK: Epidemiology of hepatocellular carcinoma. Can J Gastroenterol 14: 703-709, 2000.

2. Tung-Ping Poon R, Fan ST and Wong J: Risk factors, prevention, and management of postoperative recurrence after resection of hepatocellular carcinoma. Ann Surg 232: 10-24, 2000.

3. Brahimi-Horn MC, Chiche J and Pouyssegur J: Hypoxia and cancer. J Mol Med 85: 1301-1307, 2007.

4. Graeber TG, Osmanian C, Jacks T, et al: Hypoxia-mediated selection of cells with diminished apoptotic potential in solid tumours. Nature 379: 88-91, 1996.

5. Cavallaro U and Christofori G: Cell adhesion and signalling by cadherins and Ig-CAMs in cancer. Nat Rev Cancer 4: 118-132, 2004.

6. Choi YS, Shim YM, Kim SH, et al: Prognostic significance of E-cadherin and beta-catenin in resected stage I non-small cell lung cancer. Eur J Cardiothorac Surg 24: 441-449, 2003.

7. Bremnes RM, Veve R, Gabrielson E, et al: High-throughput tissue microarray analysis used to evaluate biology and prognostic significance of the E-cadherin pathway in non-small-cell lung cancer. J Clin Oncol 20: 2417-2428, 2002.

8. Tsujii $\mathrm{M}$ and DuBois RN: Alterations in cellular adhesion and apoptosis in epithelial cells overexpressing prostaglandin endoperoxide synthase 2. Cell 83: 493-501, 1995 .

9. Liu D, Huang C, Kameyama K, et al: E-cadherin expression associated with differentiation and prognosis in patients with non-small cell lung cancer. Ann Thorac Surg 71: 949-954, 2001.

10. Giroldi LA, Bringuier PP, de Weijert M, Jansen C, van Bokhoven A and Schalken JA: Role of $\mathrm{E}$ boxes in the repression of E-cadherin expression. Biochem Biophys Res Commun 241: 453-458, 1997.
11. Perl AK, Wilgenbus P, Dahl U, Semb H and Christofori G: A causal role for E-cadherin in the transition from adenoma to carcinoma. Nature 392: 190-193, 1998.

12. Moody SE, Perez D, Pan TC, et al: The transcriptional repressor Snail promotes mammary tumor recurrence. Cancer Cell 8: 197-209, 2005.

13. Yan W, Fu Y, Tian D, et al: PI3 kinase/Akt signaling mediates epithelial-mesenchymal transition in hypoxic hepatocellular carcinoma cells. Biochem Biophys Res Commun 382: 631-636, 2009.

14. Cannito S, Novo E, Compagnone A, et al: Redox mechanisms switch on hypoxia-dependent epithelial-mesenchymal transition in cancer cells. Carcinogenesis 29: 2267-2278, 2008.

15. Liu L, Ren ZG, Shen Y, et al: Influence of hepatic artery occlusion on tumor growth and metastatic potential in a human orthotopic hepatoma nude mouse model: relevance of epithelial-mesenchymal transition. Cancer Sci 101: 120-128, 2010.

16. Artavanis-Tsakonas S, Rand MD and Lake RJ: Notch signaling: cell fate control and signal integration in development. Science 284: 770-776, 1999.

17. Miele L and Osborne B: Arbiter of differentiation and death: Notch signaling meets apoptosis. J Cell Physiol 181: 393-409, 1999.

18. Gustafsson MV, Zheng X, Pereira T, et al: Hypoxia requires notch signaling to maintain the undifferentiated cell state. Dev Cell 9: 617-628, 2005.

19. Gao J, Song Z, Chen Y, et al: Deregulated expression of Notch receptors in human hepatocellular carcinoma. Dig Liver Dis 40: 114-121, 2008

20. Zlobin A, Jang M and Miele L: Toward the rational design of cell fate modifiers: notch signaling as a target for novel biopharmaceuticals. Curr Pharm Biotechnol 1: 83-106, 2000.

21. Jang MS, Zlobin A, Kast WM and Miele L: Notch signaling as a target in multimodality cancer therapy. Curr Opin Mol Ther 2: $55-65,2000$.

22. Sahlgren C, Gustafsson MV, Jin S, Poellinger L and Lendahl U: Notch signaling mediates hypoxia-induced tumor cell migration and invasion. Proc Natl Acad Sci USA 105: 6392-6397, 2008.

23. Lim SO, Kim HS, Quan X, et al: Notch1 binds and induces degradation of Snail in hepatocellular carcinoma. BMC Biol 9: 83, 2011.

24. Llovet JM and Bruix J: Molecular targeted therapies in hepatocellular carcinoma. Hepatology 48: 1312-1327, 2008.

25. Llovet JM, Burroughs A and Bruix J: Hepatocellular carcinoma. Lancet 362: 1907-1917, 2003.

26. Postovit LM, Adams MA, Lash GE, Heaton JP and Graham CH: Oxygen-mediated regulation of tumor cell invasiveness. Involvement of a nitric oxide signaling pathway. J Biol Chem 277: 35730-35737, 2002.

27. Rofstad EK: Microenvironment-induced cancer metastasis. Int $\mathbf{J}$ Radiat Biol 76: 589-605, 2000.

28. Lundgren K, Nordenskjold B and Landberg G: Hypoxia, Snail and incomplete epithelial-mesenchymal transition in breast cancer. $\mathrm{Br}$ J Cancer 101: 1769-1781, 2009.

29. Wells A, Yates C and Shepard CR: E-cadherin as an indicator of mesenchymal to epithelial reverting transitions during the metastatic seeding of disseminated carcinomas. Clin Exp Metastasis 25: 621-628, 2008

30. Osada T, Sakamoto M, Ino Y, et al: E-cadherin is involved in the intrahepatic metastasis of hepatocellular carcinoma. Hepatology 24: 1460-1467, 1996

31. Hotz B, Arndt M, Dullat S, Bhargava S, Buhr HJ and Hotz HG: Epithelial to mesenchymal transition: expression of the regulators snail, slug, and twist in pancreatic cancer. Clin Cancer Res 13: 4769-4776, 2007.

32. Moreno-Bueno G, Cubillo E, Sarrio D, et al: Genetic profiling of epithelial cells expressing E-cadherin repressors reveals a distinct role for Snail, Slug, and E47 factors in epithelial-mesenchymal transition. Cancer Res 66: 9543-9556, 2006.

33. Cano A, Perez-Moreno MA, Rodrigo I, et al: The transcription factor snail controls epithelial-mesenchymal transitions by repressing E-cadherin expression. Nat Cell Biol 2: 76-83, 2000.

34. Batlle E, Sancho E, Franci C, et al: The transcription factor snail is a repressor of E-cadherin gene expression in epithelial tumour cells. Nat Cell Biol 2: 84-89, 2000.

35. Yokoyama K, Kamata N, Hayashi E, et al: Reverse correlation of E-cadherin and snail expression in oral squamous cell carcinoma cells in vitro. Oral Oncol 37: 65-71, 2001.

36. Jiao W, Miyazaki K and Kitajima Y: Inverse correlation between E-cadherin and Snail expression in hepatocellular carcinoma cell lines in vitro and in vivo. Br J Cancer 86: 98-101, 2002. 
37. Balint K, Xiao M, Pinnix CC, et al: Activation of Notch1 signaling is required for beta-catenin-mediated human primary melanoma progression. J Clin Invest 115: 3166-3176, 2005.

38. Buchler P, Gazdhar A, Schubert M, et al: The Notch signaling pathway is related to neurovascular progression of pancreatic cancer. Ann Surg 242: 791-800, 2005.

39. Wang Z, Banerjee S, Li Y, Rahman KM, Zhang Y and Sarkar FH: Down-regulation of notch-1 inhibits invasion by inactivation of nuclear factor-kappaB, vascular endothelial growth factor, and matrix metalloproteinase- 9 in pancreatic cancer cells. Cancer Res 66: 2778-2784, 2006.

40. Qi R, An H, Yu Y, et al: Notch1 signaling inhibits growth of human hepatocellular carcinoma through induction of cell cycle arrest and apoptosis. Cancer Res 63: 8323-8329, 2003.

41. Dubinett SM, Sharma S, Huang M, Dohadwala M, Pold M and Mao JT: Cyclooxygenase-2 in lung cancer. Prog Exp Tumor Res 37: 138-162, 2003.

42. Dannenberg AJ and Subbaramaiah K: Targeting cyclooxygenase-2 in human neoplasia: rationale and promise. Cancer Cel 4: 431-436, 2003.

43. Brown JR and DuBois RN: Cyclooxygenase as a target in lung cancer. Clin Cancer Res 10: S4266-S4269, 2004.

44. Dannenberg AJ and Zakim D: Chemoprevention of colorectal cancer through inhibition of cyclooxygenase-2. Semin Oncol 26: 499-504, 1999

45. Huang M, Stolina M, Sharma S, et al: Non-small cell lung cancer cyclooxygenase-2-dependent regulation of cytokine balance in lymphocytes and macrophages: upregulation of interleukin 10 and down-regulation of interleukin 12 production. Cancer Res 58: 1208-1216, 1998.
46. Park K, Han S, Shin E, Kim HJ and Kim JY: Cox-2 expression on tissue microarray of breast cancer. Eur J Surg Oncol 32: 1093-1096, 2006.

47. Dohadwala M, Batra RK, Luo J, et al: Autocrine/paracrine prostaglandin E2 production by non-small cell lung cancer cells regulates matrix metalloproteinase-2 and CD44 in cyclooxygenase-2-dependent invasion. J Biol Chem 277: 50828-50833, 2002.

48. Dohadwala M, Yang SC, Luo J, et al: Cyclooxygenase-2dependent regulation of E-cadherin: prostaglandin $\mathrm{E}(2)$ induces transcriptional repressors ZEB1 and snail in non-small cell lung cancer. Cancer Res 66: 5338-5345, 2006.

49. Yeh TS, Wu CW, Hsu KW, et al: The activated Notch1 signal pathway is associated with gastric cancer progression through cyclooxygenase-2. Cancer Res 69: 5039-5048, 2009.

50. Noda M, Tatsumi Y, Tomizawa M, et al: Effects of etodolac, a selective cyclooxygenase-2 inhibitor, on the expression of E-cadherin-catenin complexes in gastrointestinal cell lines. J Gastroenterol 37: 896-904, 2002.

51. Lee DS and Moss SF: COX-2 inhibition and the prevention of gastric cancer. Digestion 74: 184-186, 2006.

52. Fujimura T, Ohta T, Oyama K, Miyashita T and Miwa K: Role of cyclooxygenase- 2 in the carcinogenesis of gastrointestinal tract cancers: a review and report of personal experience. World $\mathrm{J}$ Gastroenterol 12: 1336-1345, 2006. 